\title{
IMPROVED ROBUST SHORTEST PATHS BY PENALIZED INVESTMENTS
}

\author{
Francisco Pérez-Galarce ${ }^{1}$, Alfredo Candia-VéJar ${ }^{2, *}$, Guido Maculan ${ }^{3}$ \\ AND Nelson MaCUlan ${ }^{4}$
}

\begin{abstract}
Connectivity after disasters has become a critical problem in the management of modern cities. This comes from the need of the decision-makers to ensure urgent medical attention by providing access to health facilities and to other relevant services needed by the population. Managing congestion could help maintain some routes operative even in complex scenarios such as natural disasters, terrorist attacks, protests, or riots. Recent advances in Humanitarian Logistics have handled this problem using different modeling approaches but have principally focused on the response phase. In this paper, firstly, we propose a penalized variant of an existing mathematical model for the robust $s-t$ path problem with investments. With the aim of solving the robust several-to-one path problem with investments, and due to the high complexity of this new problem, a heuristic is proposed. Moreover, this approach allows us to improve travel times in both specific paths and in a set of routes in a systemic framework. The new problem and the proposed heuristic are illustrated by an example, which corresponds to a typical city network, that provides a concrete vision of the potential application of the framework. Lastly, some managerial insights are given by the analysis of results exhibited in the example network.
\end{abstract}

Mathematics Subject Classification. 90C47, 90-08.

Received September 4, 2020. Accepted May 29, 2021.

\section{INTRODUCTION}

Logistic operations are a fundamental component in the current globalized economy moved by supply chains. The logistics operations in disasters have given rise to a new discipline, known as Humanitarian Logistics (HL), which has had a rapid development in the last decade. A disaster produces several changes in a city, modifying infrastructure and services as well as causing injuries and deaths. In order to minimize the negative consequences, many improvements to logistics operations must be implemented and coordinated by local and national actors. Numerous review articles have described the advances in HL $[1,3,5,10,13,22,26,27,37,38,40]$. These articles have shown the impact of several disasters, giving center stage to the destruction and loss of human lives, and also presenting challenges for developing and applying HL models. In Kovacs and Moshtari [31], an analysis of various review papers about HL is presented, highlighting methodological aspects that should be considered in future studies. In the planning of pre-disaster operations, several of these papers have discussed and presented

Keywords. Traffic network design, humanitarian logistics, robust optimization, minmax regret path problem with investments, travel times, critical links.

1 Department of Computer Science, Pontificia Universidad Catolica de Chile, Santiago, Chile.

2 Department of Industrial Engineering, Faculty of Engineering, Universidad de Talca, Curicó 3340000, Chile.

3 MGN, Rio de Janeiro 20210-030, Brazil.

4 Universidade Federal do Rio de Janeiro, COPPE \& IM, Rio de Janeiro, Brazil.

${ }^{*}$ Corresponding author: acandia@utalca.cl 
models for addressing people's needs. However, there is a lack of more systemic studies supporting infrastructure investments to deal with the effects of disasters.

A disaster management system can be understood as a cycle composed of four stages: Mitigation, Preparation, Response, and Recovery [2,37,41]. Mitigation consists of the application of measures that prevent the disaster from occurring or reduce its effects if it does occur. Preparation enables communities to prepare plans for appropriate action in case of disaster. Logistical planning for aiding operations, establishing communication plans, coordinating operations, and aiding staff training are typical activities at this stage. Response involves the use of resources and emergency procedures carried out according to the previous planning, which requires the timely participation of staff and the delivery of all equipment to the disaster zone. Recovery refers to the activities covering long-term effects, such as stabilizing and restoring the damaged area.

Our interest is focused on the Mitigation phase, in which the decision-makers have the responsibility of identifying critical factors that could help reduce the effects of a disaster on the population. In this pre-disaster context, a key factor is maintaining robust connectivity between different places within a city [18]. In a disaster event, the accessibility to some specific facilities should be prioritized; for example, shelters, hospitals, and a gathering centre for distributing humanitarian aid are considered critical infrastructures. The literature has shown that after a disaster, several routes could be damaged or highly congested, interrupting vehicular travel [2]. Therefore, a careful plan in the pre-disaster phase for strengthening crowded areas is necessary.

A traditional approach to visualizing the existence of congestion in a city is by assessing the commute times between two places or areas in the city. Several studies have shown that in very congested regions, travel times have complex probability distributions [24,44]; consequently, some strategies have been developed to manage this complexity. Under crude uncertainty, an interval of travel times is adopted in the literature, and some optimization models have been defined based on this information about travel times.

Optimization with uncertainty parameters is a relevant and complex subject in Optimization. Traditionally, it includes Stochastic Optimization, in which some parameters are defined by using probability distributions [43]. Robust Optimization (RO) is a more recent research area in which some parameters are defined by using deterministic uncertainty, through uncertainty sets, for example Ben-Tal et al. [7], Gabrel et al. [20] and Kouvelis and $\mathrm{Yu}$ [30]. In particular, some studies have been published for combinatorial optimization problems with interval data $[4,12,29]$.

As a consequence of the uncertainty experienced by users when facing incremented travel times due to congestion, they need to adopt a criterion about the decision-making on the travel route between two points in a network which represents a city. The users typically hope to choose a route that takes the minimum travel time; that is, they hope to select the Shortest Path between two points (SP). In the context of a disaster in which having access to critical services like hospitals is important, routes with controlled congestion are crucial. It is well known the SP is easy to solve, since efficient algorithms exist for this problem $[17,23]$. However, the SP problem with interval data is more challenging to solve, since it is an NP-Hard problem [29]. One optimization criterion traditionally used under crude uncertainty is the Minmax Regret approach (MR). In the MR model for SP (MRP), any realization of the travel times in their interval produces a scenario. The set of scenarios represents all combinations of travel times associated with the network. Given a classic $s-t$ path in the network, its cost is compared with the optimal path under each feasible scenario. The regret of a path in a fixed scenario is the positive difference between the objective values of the path and the optimal path under each feasible scenario. The MR model seeks a compromised solution by minimizing the maximum regret.

In the context of HL, the concept of a (worst) scenario could be well illustrated when it is associated with a disaster like, for example, an earthquake. After an earthquake, even while considering some practical recommendations, people's personal or family-based decision-making is highly influenced by the magnitude of the event. Many people would likely need to get medical assistance, while others would like to visit relatives. Additionally, among other situations, some public services, like firefighters, would be activated. Hence, the massive number of vehicles and people in the streets would generate a horrible scenario (maybe the worst) in terms of the travel times for connecting the different neighbourhoods in the city. Therefore, suitable investments in infrastructure in specific places in the city in the pre-disaster phase would improve the performance in the 
transport network. Information about critical links having long travel time intervals on a typical day is key for focusing investments. For this reason, it is a basic component in the approach proposed in Section 3 when the several-to-one path problem with investments is studied. When a severe disaster occurs, an essential objective is to minimize the maximum regret over decisions related to travel routes to hospitals or shelters. It is worth mentioning that this type of objective function is coherent with the environment of HL, as some specialists in HL have revindicated $[25,31]$.

Exact and heuristic algorithms have been proposed for solving MRP, and suitable alternatives exist to solve the problem $[29,42]$. Also, note that the MR model has already been used in HL when evacuation problems were studied $[6,8,9,47]$. As an extension of the MR model, an interesting optimization model for the Minmax regret combinatorial optimization problem with investments (MRI) has been recently proposed [16]. In Conde and Leal [16], a new MR combinatorial optimization problem with interval data is presented, consisting of the addition of a new level to the classic MR problems. This new addition is an investment function on the arcs of the network to reduce the uncertainty associated with the arcs in the network. Conde and Leal [16] presented a mathematical programming formulation for the new problem so that, by applying some optimization properties and using some assumptions, the model can be solved by standard optimization packages. The authors applied the new model with investments to the MRP problem (denoted MRPI), and an illustration of the impact of the investment function was shown.

The following contributions are presented in this paper.

(i) An extension of the MRPI problem that introduces a penalty over the investment is proposed. It is shown that this problem can be utilized in HL in a pre-disaster phase. According to our knowledge, this is the first proposal for studying the application of an MR problem to improving travel times in a congested urban transport network by using effective investments in the network.

(ii) The problem defined in (i), MRPI-PEN ${ }_{a}$, is generalized to a more systemic and novel version (MRPI$\mathrm{PEN}_{O}$ ), in which the several-to-one shortest path problem is the base problem. Due to its high complexity, we propose a heuristic for finding a set of paths that includes a set of fortified arcs by the given investments. This procedure is detailed and illustrated by using a network representing a typical urban region of a city. The effectiveness of the methodology in some specific scenarios is illustrated.

(iii) A discussion about managerial insights of the model defined in (ii) helps to understand its potential application in real disasters. Some specific applications are discussed illustrating how the problems proposed can help to decision-makers to decide good investments in the network.

The paper is organized as follows. Section 2 provides an analysis of related work and Section 3 presents the optimization model, showing its variants. The heuristic for fortifying a network is presented in Section 4 . Experiments that provide a realistic application example is presented in Section 5. Lastly, conclusions and future work are presented in Section 6.

\section{LiterATURE REVIEW}

Several papers in HL have addressed problems related to connectivity during severe disasters, including some which examined the investment stage in the pre-disaster phase. In the following review, we have selected only those most related to connectivity problems, including uncertainty parameters in the associated models.

A traditional approach for tackling uncertainty in optimization models is by means of RO; in particular, this strategy has been applied to solve emergency logistics problems in transport systems. Ben-Tal et al. [7] proposed the use of RO for dynamically assigning emergency response and evacuation traffic flow problems with time-dependent demand uncertainty. They considered their problems in a humanitarian relief supply chain, and they focused on outbound emergency logistics, in which the demand for traffic flows is usually highly uncertain and depends on several factors, including the nature of the disaster and the time of impact. In order to mitigate the risk of uncertain demands, they studied the problem of generating evacuation transportation plans that are robust to uncertainty in outgoing demand. They used some instances to illustrate the value of the RO in the context of emergency logistics and to demonstrate the computational viability of the developed 
framework. Ben-Tal et al. [7] designed a RO framework for solving dynamic traffic assignment problems. The proposed RO framework was applied to the emergency response and logistics planning problems, demonstrating the computational viability of this approach. Experiments showed that the solutions provided excellent results when compared to the solutions of classic methods. Liu et al. [33] studied an optimal transport scheme that considered both the uncertainty of the multimodal transport network and the timetable limit. Due to the uncertainties of the multimodal transport network, interval data were used to represent the uncertainty of network weights; hence, RO was utilized. An optimal model of robust SP considering a timetable limit was established and a genetic algorithm was designed to solve the problem. Though this paper did not consider the context of $\mathrm{HL}$, it is mentioned here because it highlights the importance of RO in the presence of uncertainty, and it also used interval data for modelling uncertain travel times.

In order to incorporate different factors involved in the transport systems, several works have applied multiobjective or multi-criteria models to handle this complex problem. Ghavami [21] presented an integrated methodology to evaluate the transportation network performance in disaster situations by developing a multi-criteria spatial decision support system. In the transportation network studied, the identification of a set in a strategic road section was analyzed in the context of a case study. Javadian et al. [28] studied an HL network design problem, including local distribution centres and multiple central warehouses, and developed a scenario-based SP approach. The uncertainty associated with supply and demand information and the availability of the transportation network's routes after an earthquake are modelled by stochastic optimization. The proposed model attempts to minimize the total costs of the relief chain and implicitly minimize the maximum travel time between each pair of facilities and the demand point of the items. A combination of an exact and a metaheuristic approach was used to solve a real case study of a disaster that occurred in Iran. Bozorgi-Amiri et al. [11] developed a multi-objective robust SP approach for disaster relief logistics under uncertainty. In this approach, supplies, demands, and the cost of procurement and transportation were considered as uncertain parameters. They presented a case study of the robust stochastic optimization approach for disaster planning for earthquake scenarios in a region of Iran. Their findings showed that the proposed model could help in making decisions on both facility location and resource allocation in cases of disaster relief efforts. Chu and Chen [15] presented a two-stage stochastic programming model to identify the optimal protection plan that maximizes connectivity reliability for highway networks. The measure of connectivity reliability is defined as the expected number of origin-destination pair groups that can be served under all scenarios of link failure caused by a disaster. The main contribution of the model formulation is that the travel cost of alternative paths and travel demand can be simultaneously considered in the optimization. Li and Chung [32] used a RO approach to formulate the capacitated vehicle routing problem and the split delivery vehicle routing problem for a routing problem with uncertain travel times and demands in the context of disaster relief. The model considers five objective functions and a two-stage heuristic method is applied to solve the vehicle routing problem for large-scale instances. Nikoo et al. [36] presented a multi-objective optimization model for the transportation network design problem in emergencies, determining the optimal emergency response for trips with high priority in the aftermath of earthquakes. In particular, the model can also be used for identifying critical routes.

Other papers on network design and routing related to our problems are now described. Chen et al. [14] presented a state-of-the-art review specifically on the transport network design problem under uncertainty and the recently emerging development of reliability analysis in the problem. In this approach, uncertainty was managed under a stochastic framework. Several papers include uncertain components in their modelling, such as Fereiduni and Shahanaghi [19], which presented a network design model for HL; this proposal allows them to assist in the location and allocation decisions for multiple disaster periods. Vahdani et al. [46] presented a two-stage multi-objective location-routing-inventory model for HL network design under uncertainty. Tofighi et al. [45] presented a two-echelon HL network design problem involving multiple central warehouses and local distribution centres and developed a novel two-stage scenario-based possibilistic-stochastic programming. Wang and Nie [48] studied a traffic congestion problem. They proposed a two-stage location-allocation model for aiding the planning of emergency supplies for pre-positioning and post-disaster transportation. They illustrated their model with a case study on a hurricane threat in the southeastern U.S. and analyzed managerial insights 
about the pre-positioning supplies plan and the traffic control policy. Peeta et al. [39] studied a pre-disaster planning problem that seeks to strengthen a highway network whose links are subject to random failures due to a disaster. This paper highlights the fact that, in a disaster situation, the connectivity of short routes becomes a critical issue, which is also demonstrated in our work. They proposed an optimization model for the strategic planning problem in disaster management to facilitate link investment decisions to enhance disaster response.

In summation, it is interesting to note that many papers have studied different approaches to fortify transport networks. Some of the articles analyzed above have specifically studied the problem of investing in some network links in pre-disaster planning for strengthening a transport network $[18,39]$. However, they assumed knowledge of the survival probability of the links in the network while we assume a crude uncertainty under which only interval travel times are known. As is shown in Section 3, our approach has the ability to integrate conjointdecisions about links to be strengthened and the level of investments to apply to these links into just one model. This approach allows us to propose, in Section 4, a heuristic that helps decision-makers evaluate alternative improvements to a network in which crude uncertainty is considered.

\section{Problem Description And the optimization Model}

In Section 3.1, fundamental concepts from robust optimization are introduced, and then a mathematical programming formulation for the Minmax Regret Path problem with Investment (MRPI) is presented. Next, in Section 3.2, the variant MRPI with a Penalization (denoted MRPI-PEN) is given along with a mixed-integer linear programming formulation. Finally, in Section 3.3, a more systemic problem is proposed, in which the several-to-one shortest path problem is the underlying problem within the Minmax Regret criteria.

\subsection{The MRPI model}

MRPI [16] is an extension of the Minmax Regret Path Problem (MRP), see Kasperski [29], Montemanni and Gambardella [34], Montemanni et al. [35] and Pérez-Galarce et al. [42]. The MRP is defined on a digraph $G=(V, A)$ where $V$ is the set of nodes and $A$ is the set of arcs, with $|V|=n$ and $|A|=m$. $a$ is the source node and $z$ is the final node. For each $\operatorname{arc}(i, j) \in A$, two non negative numbers $c_{i j}^{-}$and $c_{i j}^{+}$are given where, $c_{i j}^{-} \leq c_{i j}^{+}$. The length (travel time in our case) can take on any real number from its uncertainty interval, $\left[c_{i j}^{-}, c_{i j}^{+}\right]$. The Cartesian product of the uncertainty intervals $\left[c_{i j}^{-}, c_{i j}^{+}\right],(i, j) \in A$, is denoted by $S$ and any element $s$ of $S$ is called a scenario. $S$ is the vector of all possible realizations of the costs of arcs. $c_{i j}^{s},(i, j) \in A$, denotes the travel time of the arc $(i, j)$ corresponding to scenario $s$.

Let $\mathbf{X} \in\{0,1\}^{|A|}$ be a binary vector such that $x_{i j}=1$ if the edge $(i, j) \in A$ belongs to an $a-z$ path $p$ of $G$ and $x_{i j}=0$, otherwise. For a given scenario $s$ and a given vector $\mathbf{X}$, the cost of the corresponding path is given by $F_{s}(\mathbf{X})=\sum_{(i, j) \in p} c_{i j}^{s}$. The classical SP for a fixed scenario $s \in S$ is:

$$
F_{s}^{*}=\min \left\{F_{s}(\mathbf{X}) \mid \mathbf{X} \in \Phi\right\},
$$

where $\Phi$ is the set of all binary vectors associated with the feasible $a-z$ paths of $G$.

For a fixed $\mathbf{X} \in \Phi$ and $s \in S$, the function $R(s, \mathbf{X})=F_{s}(\mathbf{X})-F_{s}^{*}$ is called the regret for $\mathbf{X}$ under scenario $s$. For a given $\mathbf{X} \in \Phi$, the worst-case regret or robust deviation is defined as:

$$
Z(\mathbf{X})=\max \{R(s, \mathbf{X}) \mid s \in S\} .
$$

The minmax regret version of the $a-z$ Path problem is given by:

$$
Z^{*}=\min \{Z(\mathbf{X}) \mid \mathbf{X} \in \Phi\} .
$$

In this framework, Conde and Leal [16] proposes a novel variant which considers that decision-maker can fortify the network by means of a budget. Hence, the MR objective is modified by a new set of variables, $T_{\geq 0}^{|A|}=\left(t_{i j}\right)$, which represents the investment in each arc. This set of variables is constrained by a budget $B$. 


$$
Z_{F}^{*}=\min \left\{Z(\mathbf{X}, \mathbf{T}) \mid \mathbf{X} \in \Phi, \sum_{(i, j) \in A} t_{i j} \leq B\right\} .
$$

First, Conde and Leal [16] developed the $\mathrm{MMR}_{a}-\mathrm{F}$ model in detail, and then, using the dual formulation for the inner problem (i.e., the model which finds the worst scenario), they obtained a compact formulation, $\mathrm{MRPI}_{a}$, which is presented below (see (3.1)-(3.7)). The variables $t_{i j}$ represent the amount of the given resource invested in the arc $(i, j) \in A$. The total amount of available resources of one unit that can not be exceeded. Let $u_{i}, i=1, \ldots, n$ be the dual variables.

$$
\begin{aligned}
& \min \sum_{(i, j) \in A}\left(c_{i j}^{+}-\alpha_{i j}^{+} t_{i j}\right) x_{i j}-u_{z} \\
& \sum_{\{i:(j, i) \in A\}} x_{j i}-\sum_{\{k:(k, j) \in A\}} x_{k j}= \begin{cases}1, & j=a \\
0, & j \in V \backslash\{a, z\} \\
-1, & j=z\end{cases} \\
& u_{j}-u_{i} \leq c_{i j}^{-}-\alpha_{i j}^{-} t_{i j}+\left(c_{i j}^{+}-\alpha_{i j}^{+} t_{i j}-\left(c_{i j}^{-}-\alpha_{i j}^{-} t_{i j}\right)\right) x_{i j}, \quad(i, j) \in A \\
& \sum_{(i, j) \in A} t_{i j} \leq 1 \\
& u_{a}=0 \\
& x_{i j} \in\{0,1\} \text {, } \\
& t_{i j} \geq 0 \text {, }
\end{aligned}
$$

As is detailed in Conde and Leal [16], the objective function (3.1), considering $u_{a}=0, u_{z}$ contains the optimal path cost under the worst scenario and after the investment. Constraints (3.2) define a path $a-z$ in a network flow formulation. Constraints (3.3) and (3.5) model the nested problem (Max) in the Min-Max regret objective function. To manage this, is turned into a minimization by using its dual problem, the well-known dual path flow formulation. In (3.3), the right-hand-side expression represents each arc cost, considering the worst scenario and modeling the investment. Constraint (3.4) represents the fact that the investments must respect the amount of the available resource. This model considers a linear reduction in the interval values shown in (3.8) and (3.9). These equations were used in the constraints (3.3) when the dual program of $\left(\mathrm{MMR}_{a}-\mathrm{F}\right)$ was obtained. Note also that in (3.1) and (3.3), the product of the variables $t$ and $x$ defines the model, (3.1)-(3.7), as a mixed-integer quadratic programming model. As detailed in Conde and Leal [16], the definition of a new set of variables transforms the model into a MILP (Mixed-Integer Linear Problem) formulation for the problem.

As explained in Conde and Leal [16], the positive impact of the network's investments could come from two facts. The first is the decrease in the length of the intervals associated with the arcs of the network. Thus, the network is more predictable, and also the maximum regret is decreased. The other case is when the length of the intervals remains fixed, but the upper and lower limits decrease accordingly. Equations (3.8) and (3.9) manage the cases before commented by using a linear decreasing of the intervals as a function of the investments. Therefore, it is assured that any positive investment in an arc would reduce the uncertainty in the network, maintaining $c_{i j}^{-}(t) \geq 0$.

The decision variables $t_{i j}$ manage the reduction in the upper and lower limits and the parameters $\alpha_{i j}^{+} \in \mathbb{R}^{\geq 0}$ and $\alpha_{i j}^{-} \in \mathbb{R}^{\geq 0}$ as follows:

$$
\begin{aligned}
c_{i j}^{-}(t) & =c_{i j}^{-}-\alpha_{i j}^{-} t_{i j} \\
c_{i j}^{+}(t) & =c_{i j}^{+}-\alpha_{i j}^{+} t_{i j},
\end{aligned}
$$

where $0 \leq c_{i j}^{-}(t) \leq c_{i j}^{+}(t), \forall t \in T$. Two examples are presented in Figure 1a and 1b. Note that, in particular, when $\alpha_{i j}^{+}=\alpha_{i j}^{-}, \forall(i, j) \in A$, the length of the uncertainty cost intervals remains constant, $\forall t \in T$. In the example 


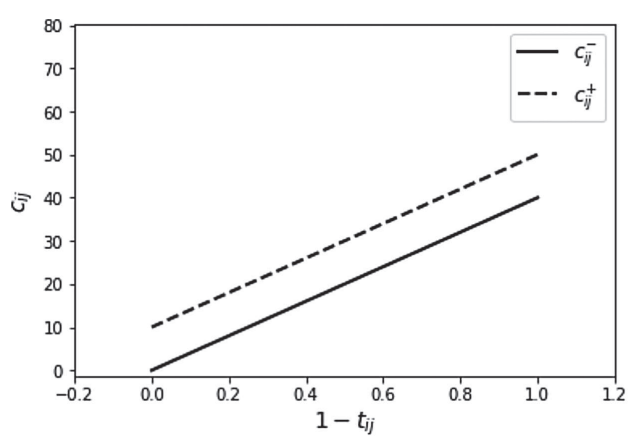

(a)

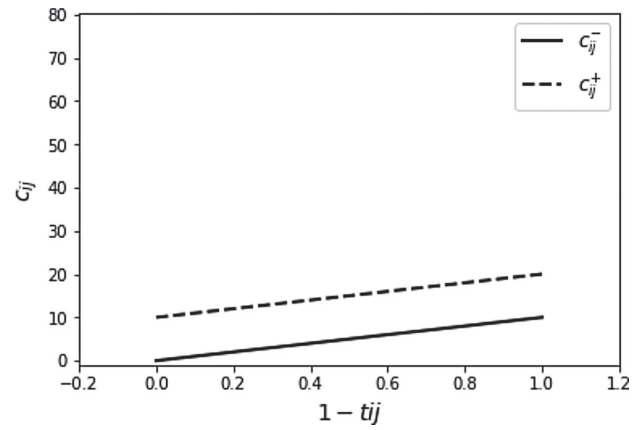

(b)

Figure 1. Profile length interval-investment for two levels of uncertainty (a) $[40,50]$ and $\alpha=40$ and (b) $[10,20]$ and $\alpha=10$.

(a) can be noted the extreme case when $t_{i j}=1$, obtaining $c_{i j}^{+}(t)=50-40 \times 1=10$ and $c_{i j}^{-}(t)=40-40 \times 1=0$. Thus, the new length interval of the improved arc under the investment is $[0,10]$. In the same way, when $t_{i j}=0.8$, the new interval is $[8,18]$. In figure $(b)$, the impact of the investment $(\alpha=10)$ is lower than the impact in the example (a) $(\alpha=40)$, so when $t_{i j}=0.8$, the new interval is $[2,12]$.

In a concrete application, these parameters $\alpha$ are defined by specialists in the field of application, who are capable of determining good estimates of the impact of the investments on the reduction of the intervals. The baseline model assumes a budget that constrains the investment but reduces the investment options in the network.

In the next section, the penalized formulation of the MRPI problem is presented.

\subsection{MRPI-PEN $a$ : A penalized MRPI problem}

The model presented in Section 3.1 is applicable when a budget for investments is known in advance. One important example is the case of infrastructure investments in a public service. In this context, standardized procedures are applied, and a specific budget could be assigned to infrastructure improvements.

However, in some real situations, principally in the context of disasters, many services are interrupted. Road connectivity, typically including bridges, walkways, paths in streets or highways, traffic lights, and other transportation infrastructure, is damaged. So, it is crucial to analyze the investments and the corresponding impacts to normalize the system's functioning. Therefore, investments should be carefully assigned so that measuring the effect of different assignments of the investments in the regret could help decision-makers in the context of a degraded system. We propose a formulation for a variant of MRPI in which the decision-maker can observe the trade-off regret-investment by means of a penalized approach. To penalize $Z_{F}^{*}$, we introduce in the objective function, the component $\lambda \sum_{(i, j) \in A} t_{i j}, \lambda \geq 0$, such that different levels of the investments (managed by the parameter $\lambda$ ) lead to different impacts in the regret of the solutions.

So, the penalized variant of $\mathrm{MRPI}_{a}$ is denoted $\mathrm{MRPI}-\mathrm{PEN}_{a}$, and its optimization model is presented in the system (3.10)-(3.16) as follows:

$$
\begin{aligned}
& \min \sum_{(i, j) \in A}\left(c_{i j}^{+}-\alpha_{i j}^{+} t_{i j}\right) x_{i j}-u_{z}+\lambda \sum_{(i, j) \in A} t_{i j} \\
& \sum_{\{i:(j, i) \in A\}} x_{j i}-\sum_{\{k:(k, j) \in A\}} x_{k j}= \begin{cases}1, & j=a \\
0, & j \in V \backslash\{a, z\} \\
-1, & j=z\end{cases} \\
& u_{j}-u_{i} \leq c_{i j}^{-}-\alpha_{i j}^{-} t_{i j}+\left(c_{i j}^{+}-\alpha_{i j}^{+} t_{i j}-\left(c_{i j}^{-}-\alpha_{i j}^{-} t_{i j}\right)\right) x_{i j}, \quad(i, j) \in A
\end{aligned}
$$




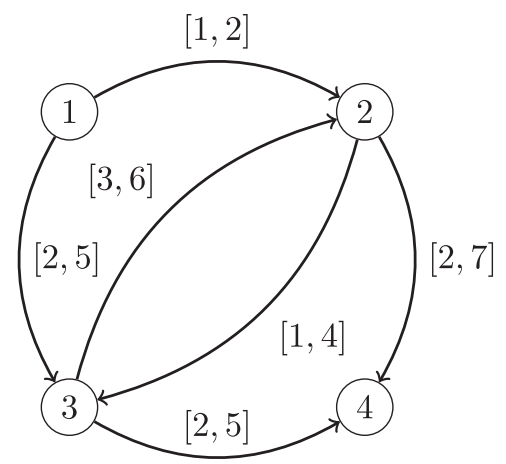

Figure 2. Example from Conde and Leal [16]. This network considers $\alpha_{i j}^{+}=\alpha_{i j}^{-}, \alpha_{12}=1$, $\alpha_{13}=4, \alpha_{23}=3, \alpha_{24}=6, \alpha_{32}=5, \alpha_{34}=4$.

$$
\begin{array}{ll}
t_{i j} \leq 1, & \forall(i, j) \in A \\
u_{a}=0 & \\
x_{i j} \in\{0,1\}, & \forall(i, j) \in A \\
t_{i j} \geq 0, & \forall(i, j) \in A .
\end{array}
$$

Note that, for $\lambda=0$, there are no limitations on the budget when the maximum regret is minimized. So, in this case, it is always possible to find an $a-z$ path such that the regret of this path is the minimum taken from all paths $a-z$, after all the possible improvements given by the equations (3.8) and (3.9). Then, when $\lambda$ is incremented, the penalized objective function limits the modifications in the arcs, and, consequently, all $a-z$ paths have a regret equal or greater than 0 when $\lambda=0$. Constraint (3.13) allows us to apply an arbitrary investment to any arc.

This model for $\mathrm{MRPI}_{a}$ offers a useful tool to assign investments under scenarios in which uncertain travel times are a crucial factor. The decision-makers can define the optimal amount of investment to provide a level of quality in the transport system (certainty in commuting times).

The example in Figure 2 considers a network from Montemanni and Gambardella [34], which was also used by Conde and Leal [16]. It has four nodes, and the arcs connecting pairs of nodes are associated with a time interval. Some values in the arcs' intervals were changed by convenience. The problem is to apply MRPI-PEN to find a 1-4 path. Different values of $\lambda$ help to find different optimal solutions with a different level of investment. In the example, the results illustrate some interesting findings, which are as follows:

- Figure 3 shows an optimal solution obtained for $\lambda=0$. The model provides a solution considering an investment equal to 1 in the arc $(1,3)$ and equal to 0.75 in the arc $(3,4)$; moreover, the optimal path $\{(1,3),(3,4)\}$ has a zero MMR value. So, since there is no penalty, the model decides to apply to the maximum possible investment to the path $(1,3),(3,4)$, and regret 0 was obtained.

- Figure 4 shows an optimal solution obtained for $\lambda=1,2,3,4,5$. The model only made an investment of 0.83 in the arc $(2,4)$ and found the optimal path $\{(1,2),(2,4)\}$ with zero MMR value. This is interesting because an investment of approximately half of that in the previous case produces a regret zero, too. This solution is also beneficial from a managerial point of view since the investment in only one arc is necessary.

The example illustrates the value of the Penalized MRPI problem modeled by the system (3.10)-(3.16) above and managed by the parameter $\lambda$. We verified that when taking any of two pairs of fortified arcs, only one path is improved in the network.

Then, the extension of the problem MRPI-PEN ${ }_{a}$ to the case when it is important to improve paths starting in different nodes and finishing in the same node $z$, defines a more systemic approach to the problem. In particular, 


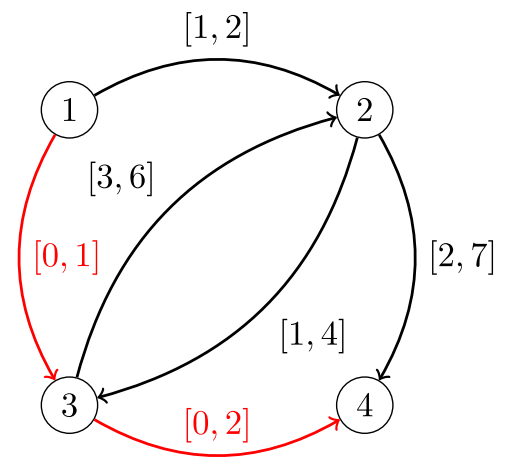

FiguRE 3. Solution for $\lambda=0 . t_{13}=1, t_{34}=0.75$. Regret $=0$, investment $=1.75$.

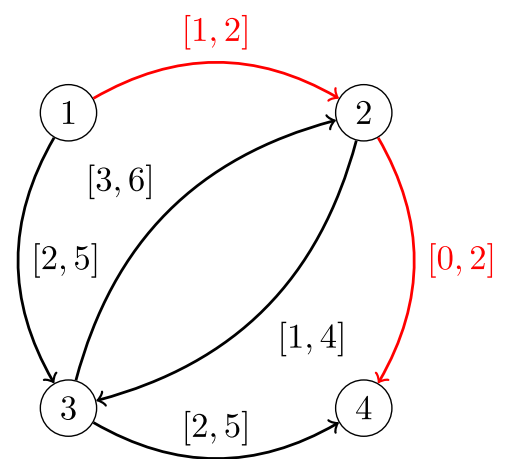

FiguRE 4. Solution for $\lambda=1-5$. Regret $=0$, investment $=0.83$.

in the field of HL, the extension is particularly important when the node $z$ represents a critical service. For example, when $z$ represents a hospital, the improvement of several paths which have $z$ as a final node would be a desirable objective.

\subsection{MRPI-PEN $O$ : Minmax regret with investments for multiple paths}

The problem MRPI-PEN ${ }_{a}$ studied above allows the assignment of investments to fortify the edges of a path $a-z$, while finding a minmax regret path in the network. However, in the context of HL, it would be important to fortify additional edges in the network so that paths starting in different nodes of $G$ can obtain improved paths to $z$. We consider that one of the paths is more critical due to some particular characteristics in the studied region. This path is called a prioritized path from now on. Additionally, to manage the difference between a prioritized path and the remaining paths, a global budget is used for the paths investments. A part of this budget is assigned to the arcs in the prioritized path, and the rest is distributed among the remaining paths.

In this context, a new problem, MRPI-PEN $O$, can be defined, which is a generalization of MRPI proposed by Conde and Leal [16]. It is assumed that a directed network $G=(V, A)$ is given, where $V$ is the set of nodes, $A$ is the set of $\operatorname{arcs},|V|=n,|A|=m, O \subseteq V$ is the set of source nodes, and $z$ is the final node. For each arc $(i, j) \in A$, two non negative numbers $c_{i j}^{-}$and $c_{i j}^{+}$are given, where $c_{i j}^{-} \leq c_{i j}^{+}$. Also, let $B$ be a budget to afford improvements in the network.

Let $\Phi^{o}$ be the set of feasible $o-z$ paths in $G, o \in O$. Given a budget $B$, MRPI-PEN $O$ seeks to find in $G=(V, A)$ a set of paths $o-z, P_{o} \in \Phi^{o}, o \in O$, and a set of binary variables $T_{\{0,1\}}^{|A|}=\left(\tau_{i j}\right) \forall(i, j) \in A$ which defines the arcs 
to be improved, such that:

$$
Z_{O}^{*}=\frac{1}{|O|} \min \left\{\sum_{o \in O} Z_{o}\left(\mathbf{P}_{\mathbf{o}}, \mathbf{T}, \lambda\right) \mid \sum_{(i, j) \in A} \tau_{i, j} \leq B, \tau_{i j} \in\{0,1\}, P_{o} \in \Phi^{o}, o \in O\right\},
$$

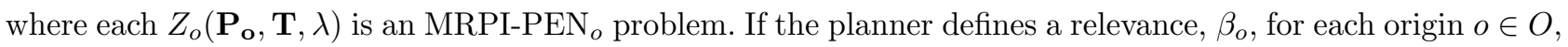
where $\sum_{o \in O} \beta_{o}=1$, we can also optimize the following weighted mean MR problem,

$$
Z_{O}^{*}=\min \left\{\sum_{o \in O} \beta_{o} Z_{o}\left(\mathbf{P}_{\mathbf{o}}, \mathbf{T}, \lambda\right) \mid \sum_{(i, j) \in A} \tau_{i, j} \leq B, \tau_{i j} \in\{0,1\}, P_{o} \in \Phi^{o}, o \in O\right\},
$$

or even define a min max problem over the set of paths as follows:

$$
Z_{O}^{*}=\min \max _{o \in O}\left\{Z_{o}\left(\mathbf{P}_{\mathbf{o}}, \mathbf{T}, \lambda\right) \mid \sum_{(i, j) \in A} \tau_{i, j} \leq B, \tau_{i j} \in\{0,1\}, P_{o} \in \Phi^{o}, o \in O\right\} .
$$

In the context of HL, each of these objective functions could be considered, and from here on, we will work with this problem (3.18).

Note that the base problem in (3.18) is a generalization of the all-to-one shortest path problem. As a result, when the minmax regret with investment is constructed, interactions between the paths in the network increment the complexity of the problem. Therefore, in order to address this complex MRPI-PEN $O$ problem, we propose

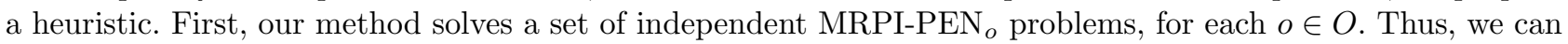
obtain a path $P_{o}$, its associated MMR value, and a set of arc investments $\psi_{i j}^{o}(\lambda)$, for a set of penalty values $\lambda \in \Lambda$. However, these results cannot be used to define a joint investment, since MRPI-PEN $O$ considers a dependent regret for each path given the investment. Additionally, the arcs found when solving each MRPI-PEN ${ }_{o}$ can be repeated. Therefore, we have to define a new second-level problem to select the arcs to be fortified after solving the set of MRPI-PEN $o$ problems. The selected arcs are defined by the variables $\tau_{i j}$ in this second-level problem. Finally, once all the investments are known, the solution of the minmax regret, for each pair $o-z$, finds the improved path.

The heuristic to address this more systemic problem is developed in the next section.

\section{A heURISTiC FOR FORTIFying NETwORKS USING MRPI-PEN}

In this section, a heuristic for solving the MRPI-PEN $O$ problem over a network is proposed, considering a flexible way to define the budget $B$ and a binary investment decision on each arc. Then, an optimization model that represents the implicit second-level problem solved by our heuristic is presented. To simplify the following explanation, without losing generality, the budget will be defined by the number of arcs in the network.

Our heuristic (pseudocode in Algorithm 1) is designed (without loss of generality) for a slight variant of MRPI-PEN $O$ which allows us to define a special source node $k$ in the network. The $k-z$ path is established as a prioritized path. In fact, if there is no a special node, we only need to apply Step 2, in which the set of paths $\mathrm{P}_{o}, \forall o \in O$ would be fortified.

\subsection{The heuristic to solve MRPI-PENO}

Input: a directed network $G=(V, A),|V|=n$ and $|A|=m . O \subseteq V$ is the set of source nodes, and $z$ is the final node, $k \in V \backslash z$. Let $B$ be the budget.

Output: let $A^{*}$ be the set of fortified arcs, $P_{k}$ is a feasible $k-z$ path, $Z^{*}$ is the regret for the several-to-one path problem, $P_{O}$ is a set of $o-z$ paths where $o \in O, Z_{O}^{*}$ is the regret value for $P_{O}$. 
Step 0. Budget initialization: let $B$ be the aforementioned budget (see (3.17)) to fortify $G$. This budget can be divided in the following two parts : (i) a prioritized $k-z$ path which can be improved $\left(B_{k}\right)$ and (ii) a systemic investment $\left(B_{O}\right)$ which can be done over $G$, where:

$B=B_{k}+B_{O}, B_{k}=\left\lfloor B * \beta_{k}\right\rfloor$, and $0 \leq \beta_{k} \leq 1$.

$\beta_{k}$ represents the proportion of the budget assigned to the $k-z$ path.

$A^{*}=\emptyset$.

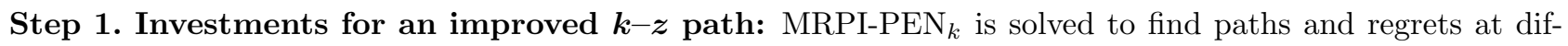
ferent levels of investment. The output in this step is, for each value of $\lambda \in \Lambda$, a set of $\operatorname{arcs} A_{k}(\lambda) \subseteq A$ in the $k-z$ path and a portfolio of investments $\psi_{i, j}^{k}(\lambda) \forall(i, j) \in A$ (see lines 6-11 in Algorithm 1). Note that it is possible to collect information about the investments assigned to each selected arc depending on $\lambda$ values (see lines 9-12 in Algorithm 1). We propose a ranking of the selected $\operatorname{arcs} \mathcal{R}_{k}$ by considering an average investment (proxy for each arc value/relevance on the network) over a set of values for $\lambda$, which is denoted by $\bar{\psi}_{i, j}^{k},(i, j) \in A$. In this way, the $B_{k}$ most important arcs are added to $A^{*}$.

Then, to obtain the fortified $k-z$ path, we implemented a procedure named $\operatorname{obtainPath}\left(A^{*}\right)$. This procedure sorts the set of paths obtained for each $\lambda \in \Lambda$, and then, considering the fortified arcs, $A^{*}$, the $k-z$ path that received the highest level of investment is selected (see line 14 in Algorithm 1). Note that in the context of a heuristic, alternatives exist in this part. For example, once the arcs selected for investments are known, a new MRP problem over the fortified network which is known could be solved.

Step 2. Investments for improved paths in $\boldsymbol{P}_{\boldsymbol{o}}$ : This step identifies the best arc-investments in the network for improving all the $o-z$ paths, $\forall o \in O$. We solve MRPI-PEN ${ }_{o}, \forall o \in O$, for different values of $\lambda$, obtaining a set of $\operatorname{arcs} A_{o}(\lambda) \subseteq A$ and a portfolio of investments $\psi_{i, j}^{o}(\lambda)(i, j) \in A$, for each $\lambda$ and source $o \in O$ (see lines 17-24 in Algorithm 1). Next, we average the investment by each $\operatorname{arc}(i, j) \in A$ and by each $\lambda \in \Lambda$. This procedure allows us to identify the $\operatorname{arcs}$ with a higher average investment impact $\bar{\psi}_{i, j}^{o},(i, j) \in A$. Lastly, based on the average values, a second ranking $\mathcal{R}_{O}$ is to fortify the first $B_{O} \operatorname{arcs}$. The selected $\operatorname{arcs}$ in this phase must be different from the fortified arcs in Step 1. Which is to say, the arcs fortified in Step 1 are deleted from the set of elements to construct the feasible solutions (arcs) (See lines 25 and 26 in Algorithm 1). Then, to obtain the fortified $o-z$ paths, $o \in O$, the paths that received the highest level of investment, for $\lambda \in \Lambda$, are selected (See line 27 in Algorithm 1).

Therefore, the heuristic in Step 1 fortifies the individual $k-z$ path, and Step 2 allows us to improve other parts of the network when a set of $o-z$ paths, $o \in O$, is fortified. Note that Steps 1 and 2 include a common procedure that generates a ranking of selected arcs to obtain the solution for MRPI-PEN ${ }_{k}$ and $\mathrm{MRPI-PEN} O$, respectively. Then, a part of these arcs is finally included in the respective solutions, depending on the particular budgets $B_{k}$ and $B_{O}$. The implicit second-level problem procedure can be modeled as the well-known $0-1$ Knapsack problem, and it is presented as follows.

\section{A $0-1$ Knapsack problem}

The specific 0-1 Knapsack problem defined in the implicit second-level problem is denoted as $\operatorname{knapsack}\left(\overline{\mathbf{\Psi}}^{k}\right.$, $\left.b, A^{*}\right)$, and it is formally defined below:

$$
\begin{aligned}
& \max \sum_{(i, j) \in A, o \in O} \beta_{o} \bar{\psi}_{i j}^{o} \tau_{i, j} \\
& \sum_{(i, j) \in A} \tau_{i j} \leq b \\
& \tau_{i, j}=0, \\
& \tau_{i, j} \in\{0,1\},
\end{aligned}
$$

where $b$ is the standard knapsack budget, depending on the step. This can represent $B_{k}$ (line 14 in Algorithm 1 ) or $B_{O}$ (line 25 in Algorithm 1). The average investment $\left(\bar{\psi}_{i j}^{o}\right)$ for each $\operatorname{arc}(i, j) \in A$ and each path $P_{o}$ is defined 
as follows:

$$
\bar{\psi}_{i j}^{o}=\sum_{\lambda \in \Lambda} \psi_{i j}^{o}(\lambda) \quad \forall(i, j) \in A .
$$

It is known that the 0-1 Knapsack is an NP-hard problem, and also it has a pseudo-polynomial algorithm based on dynamic programming. In this special case, the optimum solution of the knapsack problem is easily obtained by applying a greedy strategy, once the rankings defined in Steps 1 and 2 of the heuristic are calculated.

In Algorithm 1, only the problem MRPI-PEN ${ }_{o}$ could take significant time. However, this problem could be solved, for a fixed $\lambda \in \Lambda$, by the heuristic proposed in Conde and Leal [16], in which several computational experiments showed that the heuristic is able to solve large size instances in reasonable times. So, in lines 17 and 18 of Algorithm 1, the size of the set $O,|O|$, and $|\Lambda|$ define the time spent in this part, as a function of the times the heuristic for MRPI-PEN ${ }_{o}$ is applied. Therefore, the heuristic in Algorithm 1 could be solved in a reasonable amount of time for large sizes instances.

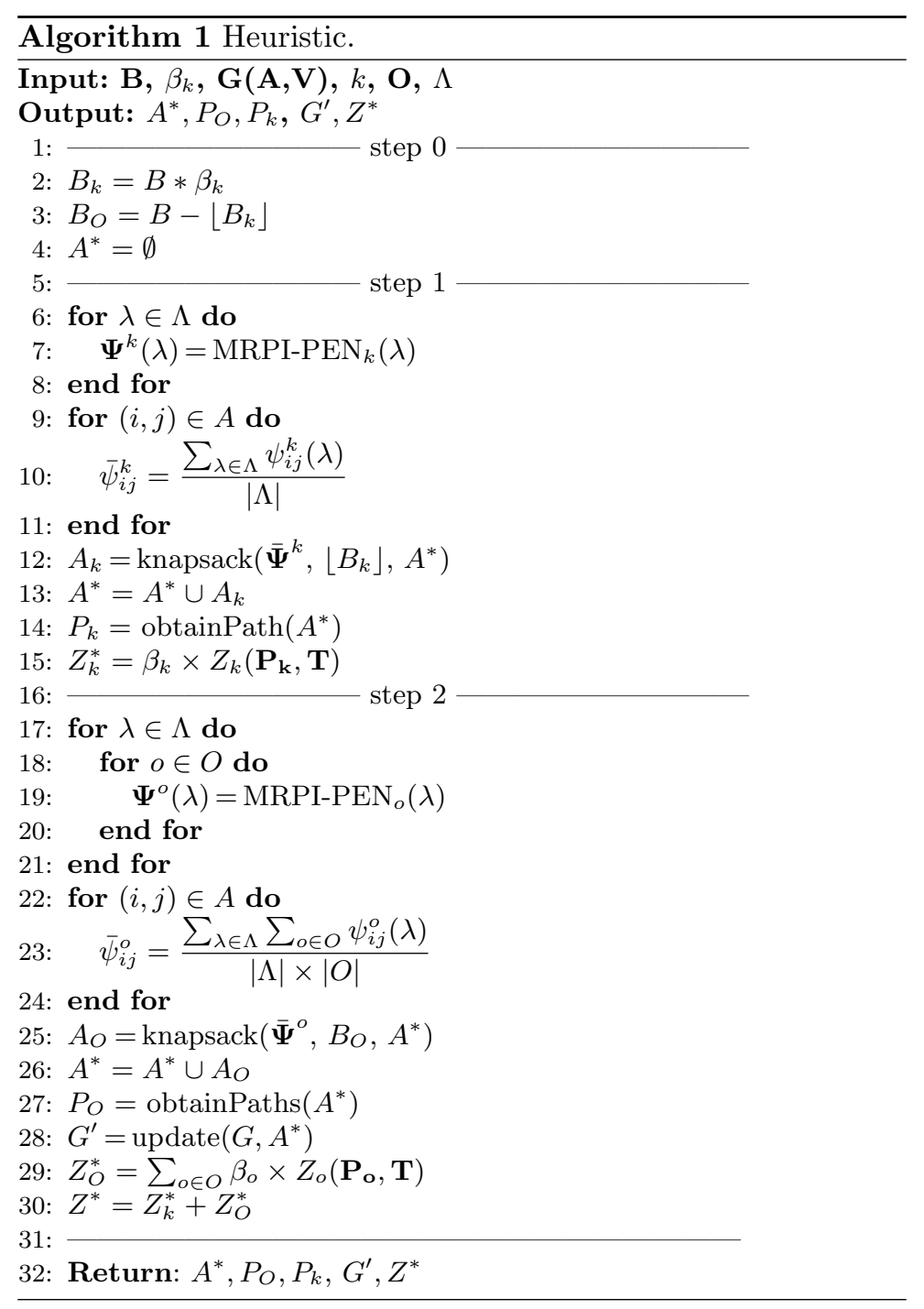




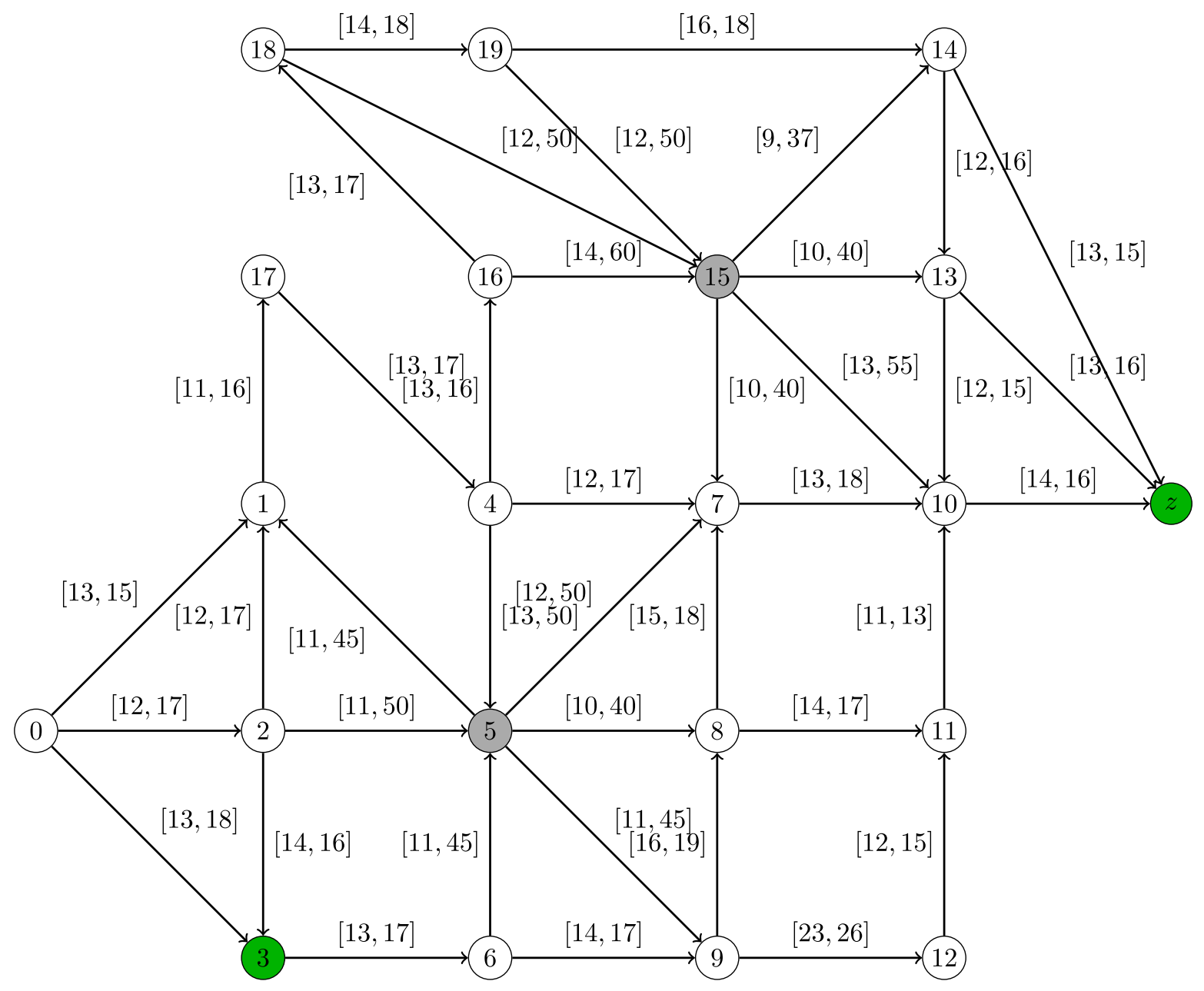

FiguRE 5. Example network in a pre-disaster scenario. Green nodes represent the source and the final nodes. Gray nodes are associated with congested zones.

\section{MRPI-PEN $k, O$ : EXPERIMENTS AND SOLUTIONS ANALYSIS}

The network in Figure 5 shows a typical scenario in which MRPI-PEN $O$ can help make decisions. Let the nodes represent zones of demand in the city and the edges denote their travel time intervals. For defining a source $(k=3)$ and a target $(z)$ in the path, two special nodes which are coloured green are considered. Target node $z$ can be associated with a hospital or a shelter. All remaining nodes form the set $O$ of source nodes, which is used in Step 2. The value of the budgets $B, B_{0}$ and $B_{k}$ are defined in Step 1. Furthermore, the network includes a subset of nodes representing highly congested zones (grey nodes), like food suppliers. The magnitude of the interval length of the arcs entering a grey node will be affected after a disaster. The congested zones add complexity to the system, since the variability in these arcs is enormous. Our approach allows us to evaluate its relevance over the network.

In the scenario presented in Figure 5, let us consider a victimized person (or a group of people) as a consequence of the disaster, who needs to go through the network from node 3 to node $z$. Good examples of this are an emergency vehicle going from an affected zone to a hospital or workers who want to return to their houses. 
TABLE 1. Results for different levels of penalization for the $\operatorname{MRPI}_{-\mathrm{PEN}_{3}}(\lambda)$ problem.

\begin{tabular}{llll}
\hline \hline$\lambda$ & Optimal solution & Regret & Investment \\
\hline 0 & $3-(1)-6-(1)-9-(1)-8-(1)-11-(1)-10-(1)-z$ & 19 & 6 \\
1 & $3-6-(1)-9-(1)-8-(1)-11-(1)-10-z$ & 19 & 4 \\
2 & $3-6-(1)-9-(1)-8-(1)-11-10-z$ & 21 & 3 \\
$3-10$ & $3-6-9-8-11-10-z$ & 30 & 0 \\
\hline
\end{tabular}

We define this actor as the commuter. Our decision tool provides a way to manage the connectivity in urban networks through an optimal investment over the arcs. As previously noted, the parameters $\alpha_{i j}^{+}$and $\alpha_{i j}^{-}$represent the expected impact due to the implementation of the set of projects (investments) on the reduction of the commuting times once the investments are applied. These projects could involve infrastructure improvements or better routes design, considering tactical or strategical investments. In the experiments, we consider $\alpha_{i j}^{+}=\alpha_{i j}^{-}$ with a value as a function of $c_{i j}^{+}$(20\% of its value). In Sections 5.1 and 5.2, we apply the heuristic for fortifying the network in Figure 5. Moreover, in Section 5.3, we provide managerial insights for the decision-makers.

\subsection{Step 1: Investments for an improved $k-z$ path}

The first step is based on impact evaluation over an individual path. In this case, we consider that the planner has given more relevance to a path from $k=3$ to $z$.

The penalized problem allows us to assess the trade-off regret investment at different levels in a scheme similar to the Pareto optimal curve. Table 1 presents different levels of penalization for the MRPI-PEN ${ }_{3}(\lambda)$ problem. The path representation $0-q-(1)-z$ considers a trail from 0 to $q$ and then to $z$, executing $100 \%$ in the improvement project $\alpha_{0 q}$, where $(0, q) \in A, q$ represents any node in the path and $q \in A \backslash\{0, z\}$. For these solutions, the regret ranges from 30 (investment $=0$ ) to 19 (investment $=6$ ). However, unlike the example in Section 3.2 , the $k-z$ path remains constant.

The main outcome in this phase is a ranking of arcs. This ranking is composed of the arcs with $\bar{\psi}_{i j}^{k} \geq 0$ for any $\lambda$ value. To generate this ranking, we aggregate the investment over the $\lambda$ values, and, after that, we divide this by the total investment $(6+4+3=13)$. For example, in Table 1 , the arc $3-6$ has a value of $(1 / 13)$ since only $\lambda=0$ considers investing in this arc. By sorting the arcs by the mean investment, we can obtain the next order [6-9 (3/13), 9-8 (3/13), 8-11 (3/13), 11-10 (2/13), 10-z (1/13), 3-6 (1/13)]. To see a variety of different solutions (path, investment, and regret) for the MRPI-PEN , $_{k}$ in Table 2 more examples of individual $k-z$ paths over the network in Figure 5 are presented.

With respect to the budget, we define $B=9$ arcs and $\beta_{k}=4 / 9$. Since $B_{k}=4$, the four selected edges are ([6-9, 9-8, 8-11, 11-10]). Hence, the budget for investing on the systemic network (Step 2) performance is $B_{o}=B-B_{k}=9-4=5$.

\subsection{Step 2: Investments for improved paths in $P_{O}$}

The second step focuses on identifying the most relevant arcs for the systemic network performance, assessing paths $o-z, o \in O$. Given that the example network is small, we take all the possible nodes in the network as a potential source node, thus, $O=V \backslash\{3, z\}$. For that, we solve MRPI-PEN M $_{k}$ for different values of $\lambda \in$ $\{0,1,2, \ldots, 9,10\}$, and then we average the investment by each arc. This procedure allows us to identify the arc with a higher average investment (see Fig. 6). With this information, we assign the resources $B_{O}$ for the $\operatorname{arcs}$ $[5-7,10-z, 15-14,2-5,19-14]\left(B_{O}=5\right)$. Lastly, in Figure 7 the fortified network is shown, and the fortified arcs are coloured in red. 
TABLE 2. Results of different $k-z$-paths and levels of penalization over MRPI-PEN ${ }_{k}$.

\begin{tabular}{lllll}
\hline \hline Source & $\lambda$ & Optimal solution & Regret & Investment \\
\hline 0 & 0 & $0-(1)-1-(1)-17-(1)-4-(1)-7-10-(1)-z$ & 18 & 5 \\
& $1-2$ & $0-(1)-1-(1)-17-(1)-4-(1)-7-10-z$ & 18 & 4 \\
& $3-10$ & $0-1-17-4-7-10-z$ & 30 & 0 \\
\hline 18 & 0 & $18-(1)-19-(1)-14-(1)-z$ & 9 & 3 \\
& $1-2$ & $18-(1)-19-(1)-14-(0 . \overline{3})-z$ & 9 & $2 . \overline{3}$ \\
& $3-10$ & $18-19-14-z$ & 16 & 0 \\
\hline 17 & 0 & $17-(1)-4-(1)-7-10-(1)-z$ & 0 & 3 \\
& $1-2$ & $17-4-7-(0 . \overline{3})-10-(0 . \overline{3})-z$ & 0 & $0 . \overline{6}$ \\
& $3-10$ & $17-4-7-10-z$ & 2 & 0 \\
\hline 5 & $0-2$ & $5-(1)-7-(1)-10-z$ & 20 & 2 \\
& $3-9$ & $5-(1)-7-10-z$ & 23 & 1 \\
& 10 & $5-7-10-z$ & 33 & 0 \\
\hline 15 & $0-2$ & $15-(1)-14-(1)-z$ & 19 & 2 \\
& $3-6$ & $15-(1)-14-z$ & 22 & 1 \\
& $7-10$ & $15-14-z$ & 29 & 0 \\
\hline
\end{tabular}

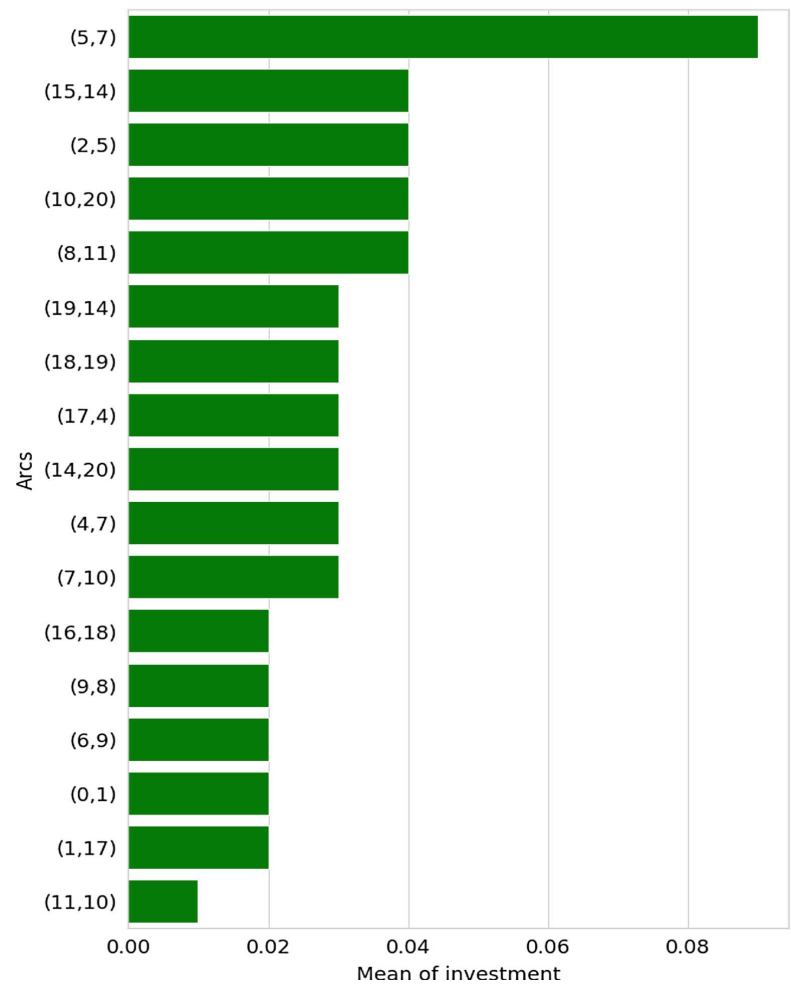

FiguRE 6. Average investment $\bar{\psi}_{i j}^{o}$ for the most important arcs.

The obtained solutions have the following values (Algorithm 1 line 30):

$$
Z^{*}=\frac{4}{9} \times Z_{k}\left(\mathbf{P}_{\mathbf{k}}^{*}, \mathbf{T}^{*}\right)+\frac{\frac{5}{9}}{19} \times \sum_{o \in O \backslash k} Z_{o}\left(\mathbf{P}_{\mathbf{o}}^{*}, \mathbf{T}^{*}\right)
$$




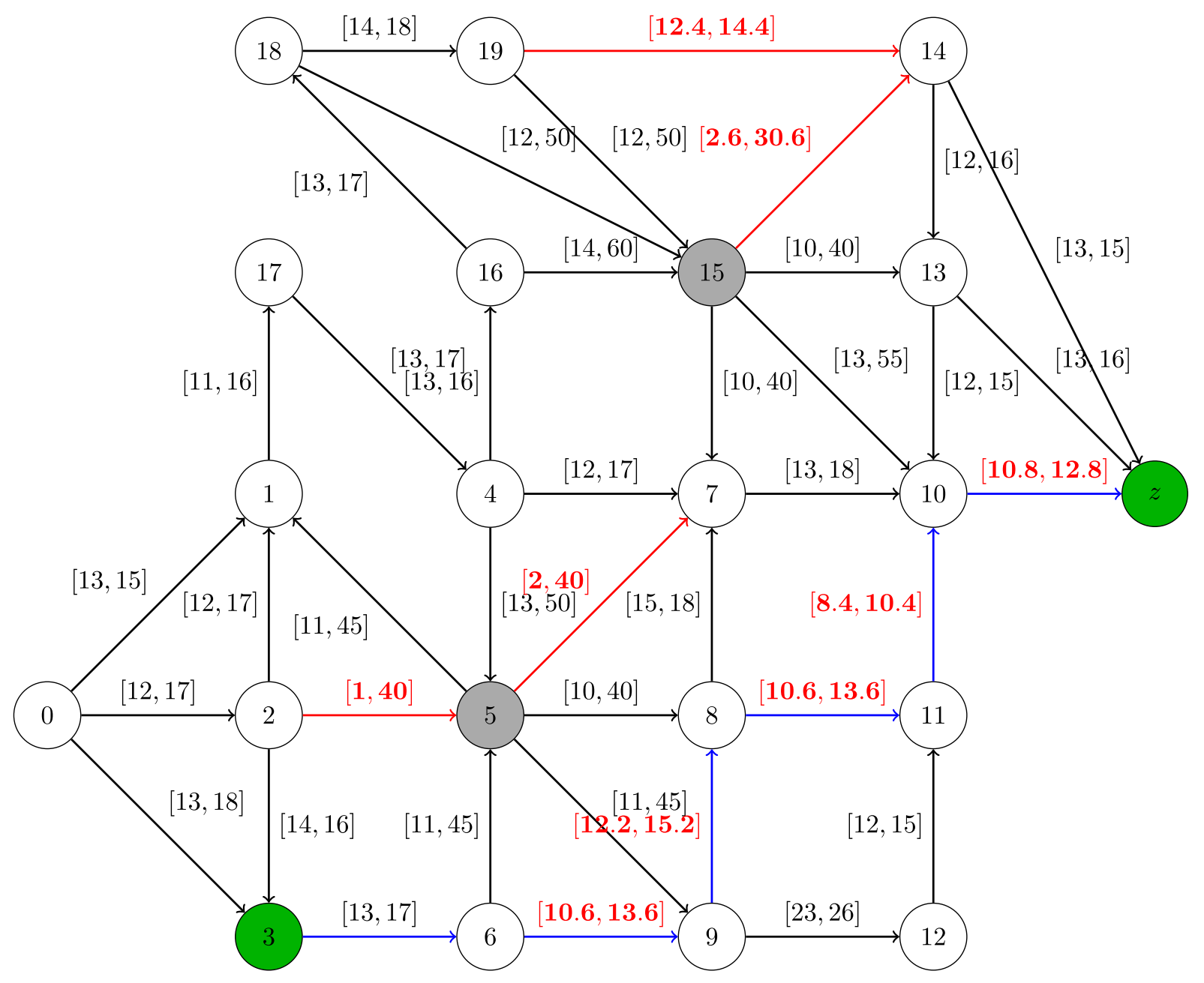

FIGURE 7 . Black arcs represent the $k-z$ path, and red intervals represent travel times after an investment (fortified arcs).

$$
\begin{aligned}
& Z^{*}=\frac{4}{9} \times 30+\frac{\frac{5}{9}}{19} \times 223=19.85 \\
& Z^{*}=\frac{4}{9} \times 25+\frac{\frac{5}{9}}{19} \times 139=15.05 .
\end{aligned}
$$

(without investment)

(with investment)

This solution reduced by $24.18 \%$ the systemic regret with respect to the model without investments. Regarding the $k-z$ path the reduction is $16 . \overline{6} \%$.

\subsection{Managerial insights}

The proposed models have suitable features for application in places where disasters are frequent. Our algorithm could be used in a decision support system to help decision-makers when implementing fortification plans. Furthermore, this approach can be used in several decision problems in which the uncertainty can be 
mitigated using investments. In smart cities, this could help to reduce the impact of terrorist attacks, riots or accidents. This heuristic also could be useful in coastal cities to minimize the uncertainty during evacuations after tsunamis. Lastly, as was noted throughout the article, it can be applied to mitigate the uncertainty of commuting times in traffic networks.

Our heuristic provides a mechanism to decision-makers to prioritize arcs under crude uncertainty beyond individual paths. It focuses on fortifying the connectivity of the network and improving the worst scenarios for more commuters. The investment plan includes improvements to mitigate a more informative regret on networks. Given that, the planner should consider more than one budget distribution to study the impact on the transport network.

In our example, it is interesting to analyze the source node 5 which represents a congested zone (similar to node 15). Any arc exiting this node has associated its time interval with very high uncertainty. Thus, at least one arc in the path $5-z$ will contain one with this property. Concretely, arc $(5,7)$ belongs to the optimal solution obtained when solving MRPI-PEN 5 , as shown in Table 2. Moreover, among all the arcs selected after solving the problems MRPI-PEN ${ }_{k}$, this arc presents the highest average investment, as shown in Figure 4. The original time interval of $(5,7)$ is $[12,50]$ and the invested arc has time interval $[2,40]$.

\section{COnClusions And FUture WORK}

Path connectivity problems in networks are fundamental in Combinatorial Optimization due to the richness of existing variants at different levels of complexity and the broad range of applications in real network problems. In this context, the present paper analyzes a robust version, which is known as the minmax regret shortest path with investments. This version adds a component of arc investments so that the solution to this problem improves the performance of the network in terms of the uncertainty of the paths. Our contribution extends this problem by incorporating a cost penalty as a component in the objective function of a MILP formulation of the problem. This model offers a way to manage a trade-off between arc-investments and the value of the minmax regret solution. Thus, this provides the decision-maker with the possibility of exploring a set of solutions.

The other contribution is the extension of the MRPI problem, in which only one source node is considered (one-to-one shortest path problem) to the same problem in which a set of source nodes are considered (a particular case is all-to-one shortest path). Due to the high complexity of this new problem, we design a heuristic that takes advantage of the information achieved after solving individual applications of the one-to-one version of the problem.

Future work includes methodological aspects such as formulating a multi-objective optimization model, including arc-investments (costs), the minmax regret solution's value, and some additional characteristics of the network, like the number of arcs in the solution. Additionally, the development of similar or alternative strategies to strengthen a network using the minmax regret model applied to other important network optimization problems, like the traveling salesman and routing problems, would be a good challenge. Even though the problems discussed in this paper are potentially applicable to several contexts in which uncertainty exists, applications in HL are essential, because most disasters provoke disruptions in the connectivity of a city. Hence, investments in infrastructure which help to mitigate the effects of a disaster are essential. In summation, the minimax regret problem with investments in the system deserves future studies beyond this paper.

Acknowledgements. F. Pérez-Galarce acknowledges the support from National Agency for Research and Development (ANID), through Scholarship Program/Doctorado Nacional/2017-21171036. N. Maculan acknowledges the support from agency CNPq and Fundação COPPETEC.

\section{REFERENCES}

[1] H. Abidi, S. de Leeuw and M. Klumpp, Humanitarian supply chain performance management: a systematic literature review. Supply Chain Manage. Int. J. 19 (2014) 592-608.

[2] M. Ahmadi, A. Seifi and B. Tootooni, A humanitarian logistics model for disaster relief operation considering network failure and standard relief time: a case study on San Francisco district. Transp. Res. Part E: Logist. Transp. Rev. 75 (2015) 145-163. 
[3] A. Ahmadi-Javid, P. Seyedi and S. Syam, A survey of healthcare facility location. Comput. Oper. Res. 79 (2017) $223-263$.

[4] H. Aissi, C. Bazgan and D. Vanderpooten, Min-max and min-max regret versions of combinatorial optimization problems: a survey. Eur. J. Oper. Res. 197 (2009) 427-438.

[5] R. Banomyong, P. Varadejsatitwong and R. Oloruntoba, A systematic review of humanitarian operations, humanitarian logistics and humanitarian supply chain performance literature 2005 to 2016. Ann. Oper. Res. 283 (2019) 71-86.

[6] R. Benkoczi, B. Bhattacharya, Y. Higashikawa, T. Kameda and N. Katoh, Minmax-regret evacuation planning for cycle networks. In: International Conference on Theory and Applications of Models of Computation. Springer (2019) $42-58$.

[7] A. Ben-Tal, B. Do Chung, S. Mandala and T. Yao, Robust optimization for emergency logistics planning: risk mitigation in humanitarian relief supply chains. Transp. Res. Part B Methodol. 45 (2011) 1177-1189.

[8] B. Bhattacharya and T. Kameda, Improved algorithms for computing minmax regret sinks on dynamic path and tree networks. Theor. Comput. Sci. 607 (2015) 411-425.

[9] B. Bhattacharya, Y. Higashikawa, T. Kameda and N. Katoh, Minmax regret 1-sink for aggregate evacuation time on path networks. Preprint arXiv:1806.00814 (2018).

[10] C. Boonmee, M. Arimura and T. Asada, Facility location optimization model for emergency humanitarian logistics. Int. J. Disaster Risk Reduct. 24 (2017) 485-498.

[11] A. Bozorgi-Amiri, M. Jabalameli and S. Al-e Hashem, A multi-objective robust stochastic programming model for disaster relief logistics under uncertainty. OR Spectr. 35 (2013) 905-933.

[12] A. Candia-Vejar, E. Alvarez-Miranda and N. Maculan, Minmax regret combinatorial optimization problems: an algorithmic perspective. RAIRO: OR 45 (2011) 101-129.

[13] A. Caunhye, X. Nie and S. Pokharel, Optimization models in emergency logistics: a literature review. Socio-Econ. Plan. Sci. 46 (2012) 4-13.

[14] A. Chen, Z. Zhou, P. Chootinan, S. Ryu, C. Yang and S. Wong, Transport network design problem under uncertainty: a review and new developments. Transp. Rev. 31 (2011) 743-768.

[15] J. Chu and S. Chen, Optimization of transportation-infrastructure-system protection considering weighted connectivity reliability. J. Infrastruct. Syst. 22 (2015) 04015008.

[16] E. Conde and M. Leal, Minmax regret combinatorial optimization problems with investments. Comput. Oper. Res. 85 (2017) $1-11$.

[17] E. Dijkstra, A note on two problems in connexion with graphs. Numer. Math. 1 (1959) 269-271. .

[18] L. Du and S. Peeta, A stochastic optimization model to reduce expected post-disaster response time through pre-disaster investment decisions. Networks Spatial Econ. 14 (2014) 271-295.

[19] M. Fereiduni and K. Shahanaghi, A robust optimization model for distribution and evacuation in the disaster response phase. J. Ind. Eng. Int. 13 (2017) 117-141.

[20] V. Gabrel, C. Murat and A. Thiele, Recent advances in robust optimization: an overview. Eur. J. Oper. Res. 235 (2014) 471-483.

[21] S. Ghavami, Multi-criteria spatial decision support system for identifying strategic roads in disaster situations. Int. J. Crit. Infrastruct. Prot. 24 (2019) 23-36.

[22] W. Gutjahr and P. Nolz, Multicriteria optimization in humanitarian aid. Eur. J. Oper. Res. 252 (2016) 351-366.

[23] P. Hart, N.J. Nilsson and B. Raphael, A formal basis for the heuristic determination of minimum cost paths. IEEE Trans. Syst. Sci. Cybern. 4 (1968) 100-107.

[24] C. Higgins, M.N. Sweet and P. Kanaroglou, All minutes are not equal: travel time and the effects of congestion on commute satisfaction in canadian cities. Transportation 45 (2018) 1249-1268.

[25] J. Holguín-Veras, N. Pérez, M. Jaller, L. Van Wassenhove and F. Aros-Vera, On the appropriate objective function for postdisaster humanitarian logistics models. J. Oper. Manage. 31 (2013) 262-280.

[26] M. Hoyos, R. Morales and R. Akhavan-Tabatabaei, Or models with stochastic components in disaster operations management: a literature survey. Comput. Ind. Eng. 82 (2015) 183-197.

[27] C.J.C. Jabbour, V.A. Sobreiro, A.B.L. de Sousa Jabbour, L.M. de Souza Campos, E.B. Mariano and D.W.S. Renwick, An analysis of the literature on humanitarian logistics and supply chain management: paving the way for future studies. Ann. Oper. Res. 283 (2019) 289-307.

[28] N. Javadian, S. Modarres and A. Bozorgi, A bi-objective stochastic optimization model for humanitarian relief chain by using evolutionary algorithms. Int. J. Eng. Trans. A: Basics 30 (2017) 1526-1537.

[29] A. Kasperski, Discrete Optimization with Interval Data. In: Vol. 228 of Studies in Fuzziness and Soft Computing. Springer Berlin Heidelberg, Berlin, Heidelberg (2008).

[30] P. Kouvelis and G. Yu, Robust Discrete Optimization and its Applications. Kluwer Academic Pablishers (1997).

[31] G. Kovacs and M. Moshtari, A roadmap for higher research quality in humanitarian operations: a methodological perspective. Eur. J. Oper. Res. 276 (2019) 395-408.

[32] Y. Li and S. Chung, Disaster relief routing under uncertainty: a robust optimization approach. IISE Trans. 51 (2019) 869-886.

[33] S. Liu, Y. Peng, Q. Song and Y. Zhong, The robust shortest path problem for multimodal transportation considering timetable with interval data. Syst. Sci. Control Eng. 6 (2018) 68-78.

[34] R. Montemanni and L. Gambardella, An exact algorithm for the robust shortest path problem with interval data. Comput. Oper. Res. 31 (2004) 1667-1680.

[35] R. Montemanni, L. Gambardella and A. Donati, A branch and bound algorithm for the robust shortest path problem with interval data. Oper. Res. Lett. 32 (2004) 225-232. 
[36] N. Nikoo, M. Babaei and A. Mohaymany, Emergency transportation network design problem: identification and evaluation of disaster response routes. Int. J. Disaster Risk Reduct. 27 (2018) 7-20.

[37] M. Ortuño, P. Cristóbal, J. Ferrer, F. Martín-Campo, S. Muñoz, G. Tirado and B. Vitoriano, Decision aid models and systems for humanitarian logistics. A survey. In: Decision Aid Models for Disaster Management and Emergencies. Springer (2013) $17-44$.

[38] L. Özdamar and M. Ertem, Models, solutions and enabling technologies in humanitarian logistics. Eur. J. Oper. Res. 244 (2015) 55-65.

[39] S. Peeta, F. Salman, D. Gunnec and K. Viswanath, Pre-disaster investment decisions for strengthening a highway network. Comput. Oper. Res. 37 (2010) 1708-1719.

[40] E. Peres, I. Brito, A. Leiras and H. Yoshizaki, Humanitarian logistics and disaster relief research: trends, applications, and future research directions. In: Proceedings of the 4th International Conference on Information Systems, Logistics and Supply Chain (2012) 26-29.

[41] F. Pérez-Galarce, L. Canales, C. Vergara and A. Candia-Véjar, An optimization model for the location of disaster refuges. Socio-Econ. Plan. Sci. 59 (2017) 56-66.

[42] F. Pérez-Galarce, A. Candia-Véjar, C. Astudillo and M. Bardeen, Algorithms for the minmax regret path problem with interval data. Inf. Sci. 462 (2018) 218-241.

[43] A. Ruszczyński and A. Shapiro, Stochastic programming models. In: Vol. 10 of Handbooks in Operations Research and Management Science (2003) 1-64.

[44] C. Shi, B. Chen and Q. Li, Estimation of travel time distributions in urban road networks using low-frequency floating car data. ISPRS Int. J. Geo-Inf. 6 (2017) 253.

[45] S. Tofighi, S. Torabi and S. Mansouri, Humanitarian logistics network design under mixed uncertainty. Eur. J. Oper. Res. 250 (2016) 239-250.

[46] B. Vahdani, D. Veysmoradi, F. Noori and F. Mansour, Two-stage multi-objective location-routing-inventory model for humanitarian logistics network design under uncertainty. Int. J. Disaster Risk Reduct. 27 (2018) 290-306.

[47] H. Wang, Minmax regret 1-facility location on uncertain path networks. Eur. J. Oper. Res. 239 (2014) $636-643$.

[48] Q. Wang and X. Nie, A stochastic programming model for emergency supply planning considering traffic congestion. IISE Trans. 51 (2019) 910-920. 